\title{
Linear codes of 2-designs associated with subcodes of the ternary generalized Reed-Muller codes
}

\author{
Cunsheng Ding ${ }^{\mathrm{a}}$, Chunming Tang ${ }^{\mathrm{b}}$, Vladimir D. Tonchev ${ }^{\mathrm{c}}$ \\ ${ }^{a}$ Department of Computer Science and Engineering, The Hong Kong University of Science and Technology, Clear \\ Water Bay, Kowloon, Hong Kong, China \\ ${ }^{b}$ School of Mathematics and Information, China West Normal University, Nanchong, Sichuan, 637002, China \\ ${ }^{c}$ Department of Mathematical Sciences, Michigan Technological University, Houghton, Michigan 49931, USA
}

\begin{abstract}
In this paper, the 3-rank of the incidence matrices of 2-designs supported by the minimum weight codewords in a family of ternary linear codes considered in [C. Ding, C. Li, Infinite families of 2-designs and 3-designs from linear codes, Discrete Mathematics 340(10) (2017) 2415-2431] are computed. A lower bound on the minimum distance of the ternary codes spanned by the incidence matrices of these designs is derived, and it is proved that the codes are subcodes of the 4th order generalized Reed-Muller codes.
\end{abstract}

Keywords: Cyclic code, linear code, Reed-Muller code, $t$-design.

2000 MSC: 05B05, 51E10, 94B15

\section{Introduction}

Let $\mathcal{P}$ be a set of $v \geq 1$ elements, and let $\mathcal{B}$ be a set of $k$-subsets of $\mathcal{P}$, where $k$ is a positive integer with $1 \leq k \leq v$. Let $t$ be a positive integer with $t \leq k$. The pair $\mathbb{D}=(\mathscr{P}, \mathcal{B})$ is called a $t$ - $(v, k, \lambda)$ design, or simply $t$-design, if every $t$-subset of $\mathcal{P}$ is contained in exactly $\lambda$ elements of $\mathcal{B}$. The elements of $\mathcal{P}$ are called points, and those of $\mathcal{B}$ are referred to as blocks. We usually use $b$ to denote the number of blocks in $\mathcal{B}$. A $t$-design is called simple if $\mathcal{B}$ does not contain any repeated blocks. In this paper, we consider only simple $t$-designs with $v>k>t$. A $t-(v, k, \lambda)$ design is referred to as a Steiner system if $t \geq 2$ and $\lambda=1$, and is denoted by $S(t, k, v)$.

Let $\mathbb{D}=(\mathscr{P}, \mathcal{B})$ be a $t-(v, k, \lambda)$ design with $b \geq 1$ blocks. The points of $\mathscr{P}$ are usually indexed with $p_{1}, p_{2}, \cdots, p_{v}$, and the blocks of $\mathcal{B}$ are normally denoted by $B_{1}, B_{2}, \cdots, B_{b}$. The incidence matrix $M_{\mathbb{D}}=\left(m_{i j}\right)$ of $\mathbb{D}$ is a $b \times v$ matrix where $m_{i j}=1$ if $p_{j}$ is on $B_{i}$ and $m_{i j}=0$ otherwise. The $p$-rank of a design $\mathbb{D}$ is defined as the rank of its incidence matrix over a finite field of characteristic $p$. The binary matrix $M_{\mathbb{D}}$ can be viewed as a matrix over $\operatorname{GF}(q)$ for any prime power $q$, and its row vectors span a linear code of length $v$ over $\operatorname{GF}(q)$, which is denoted by $\mathrm{C}_{q}(\mathbb{D})$ and called the code of $\mathbb{D}$ over $\operatorname{GF}(q)$. It is known that the $p$-rank of a $t$ - $(v, k, \lambda)$ design with $t \geq 2$ can be smaller than

\footnotetext{
${ }^{2}$ C. Ding's research was supported by the Hong Kong Research Grants Council, Proj. No. 16300418. C. Tang was supported by National Natural Science Foundation of China (Grant No. 11871058) and China West Normal University (14E013, CXTD2014-4 and the Meritocracy Research Funds).

Email addresses: cding@ust.hk (Cunsheng Ding), tangchunmingmath@163.com (Chunming Tang), tonchev@mtu.edu (Vladimir D. Tonchev)
} 
$v-1$ (hence, the dimension of $\mathrm{C}_{q}(\mathbb{D})$, where $q=p^{t}$, can be smaller than $v-1$ ), only if $p$ divides $\lambda_{1}-\lambda_{2}$, where $\lambda_{i}$ denotes the number of blocks of $\mathbb{D}$ that contain $i$ points $(i=1,2)$ (cf. [13], [26, Theorem 1.86, page 686].)

We assume that the reader is familiar with the basics of linear codes and cyclic codes. Throughout this paper, we denote the dual code of $\mathrm{C}$ by $\mathrm{C}^{\perp}$, and the extended code of $\mathrm{C}$ by $\overline{\mathrm{C}}$.

Let $\mathrm{C}$ be a $[v, \kappa, d]$ linear code over $\mathrm{GF}(q)$. Let $A_{i}:=A_{i}(\mathrm{C})$, which denotes the number of codewords with Hamming weight $i$ in $\mathrm{C}$, where $0 \leq i \leq v$. The sequence $\left(A_{0}, A_{1}, \cdots, A_{v}\right)$ is called the weight distribution of $\mathrm{C}$, and $\sum_{i=0}^{v} A_{i} z^{i}$ is referred to as the weight enumerator of $\mathrm{C}$. For each $k$ with $A_{k} \neq 0$, let $\mathcal{B}_{k}$ denote the set of the supports of all codewords with Hamming weight $k$ in $C$, where the coordinates of a codeword are indexed by $\left(p_{1}, \ldots, p_{v}\right)$. Let $\mathcal{P}=\left\{p_{1}, \ldots, p_{v}\right\}$. The pair $\left(\mathcal{P}, \mathcal{B}_{k}\right)$ may be a $t-(v, k, \lambda)$ design for some positive integer $\lambda$, which is called a support design of the code, and is denoted by $\mathbb{D}_{k}(\mathrm{C})$. In such a case, we say that the code $\mathrm{C}$ holds a $t$ - $(v, k, \lambda)$ design 1 .

While most linear codes over finite fields do not hold $t$-designs, some linear codes do hold $t$ designs for $t \geq 1$. Studying the linear codes of $t$-designs has been a topic of research for a long time $[1,3,2,5,8,14,17,22,23,26]$. The objective of this paper is to study the linear codes of a family of 2-designs held in a class of ternary linear codes. It will be shown that these codes are subcodes of the fourth-order generalized Reed-Muller codes and support new 2-designs.

\section{Auxiliary results}

In this section, we present some auxiliary results that will be needed in later sections.

\subsection{Designs from linear codes via the Assmus-Mattson Theorem}

The following theorem, proved by Assumus and Mattson [2], shows that the pair $\left(\mathcal{P}, \mathcal{B}_{k}\right)$ defined by a linear code is a $t$-design under certain conditions.

Theorem 1 (Assmus-Mattson Theorem). ([2]], [14, p. 303]) Let C be a $[v, k, d]$ code over $\mathrm{GF}(q)$. Let $d^{\perp}$ denote the minimum distance of $\mathrm{C}^{\perp}$. Let $w$ be the largest integer satisfying $w \leq v$ and

$$
w-\left\lfloor\frac{w+q-2}{q-1}\right\rfloor<d
$$

Define $w^{\perp}$ analogously using $d^{\perp}$. Let $\left(A_{i}\right)_{i=0}^{v}$ and $\left(A_{i}^{\perp}\right)_{i=0}^{v}$ denote the weight distribution of $\mathrm{C}$ and $\mathrm{C}^{\perp}$, respectively. Fix a positive integer $t$ with $t<d$, and let $s$ be the number of $i$ with $A_{i}^{\perp} \neq 0$ for $0 \leq i \leq v-t$. Suppose $s \leq d-t$. Then

- the codewords of weight $i$ in C hold a $t$-design provided $A_{i} \neq 0$ and $d \leq i \leq w$, and

- the codewords of weight $i$ in $C^{\perp}$ hold a t-design provided $A_{i}^{\perp} \neq 0$ and $d^{\perp} \leq i \leq \min \{v-$ $\left.t, w^{\perp}\right\}$.

The Assmus-Mattson Theorem is a very useful tool for constructing $t$-designs from linear codes, and has been successfully employed to construct infinitely many 2-designs and 3-designs [9].

\footnotetext{
${ }^{1}$ More generally, a code $\mathrm{C}$ holds (or supports) a $t-v, k, \lambda$ ) design $\mathbb{D}$ if every block of $\mathbb{D}$ is the support of some codeword of C [23].
} 


\subsection{Designs from linear codes via the automorphism group}

The set of coordinate permutations that map a code $\mathrm{C}$ to itself forms a group, which is referred to as the permutation automorphism group of $\mathrm{C}$ and denoted by $\operatorname{PAut}(\mathrm{C})$. If $\mathrm{C}$ is a code of length $n$, then PAut (C) is a subgroup of the symmetric group $\operatorname{Sym}_{n}$.

A monomial matrix over $\mathrm{GF}(q)$ is a square matrix having exactly one nonzero element of $\operatorname{GF}(q)$ in each row and column. A monomial matrix $M$ can be written either in the form $D P$ or the form $P D_{1}$, where $D$ and $D_{1}$ are diagonal matrices and $P$ is a permutation matrix.

The set of monomial matrices that map $C$ to itself forms the group $\operatorname{MAut}(\mathrm{C})$, which is called the monomial automorphism group of C. Clearly, we have

$$
\operatorname{PAut}(\mathrm{C}) \subseteq \operatorname{MAut}(\mathrm{C}) .
$$

The automorphism group of $\mathrm{C}$, denoted by $\operatorname{Aut}(\mathrm{C})$, is the set of maps of the form $M \gamma$, where $M$ is a monomial matrix and $\gamma$ is a field automorphism, that map $C$ to itself. In the binary case, $\operatorname{PAut}(\mathrm{C}), \operatorname{MAut}(\mathrm{C})$ and $\operatorname{Aut}(\mathrm{C})$ are the same. If $q$ is a prime, $\operatorname{MAut}(\mathrm{C})$ and $\operatorname{Aut}(\mathrm{C})$ are identical. In general, we have

$$
\operatorname{PAut}(\mathrm{C}) \subseteq \operatorname{MAut}(\mathrm{C}) \subseteq \operatorname{Aut}(\mathrm{C}) .
$$

By definition, every element in $\operatorname{Aut}(\mathrm{C})$ is of the form $D P \gamma$, where $D$ is a diagonal matrix, $P$ is a permutation matrix, and $\gamma$ is an automorphism of $\operatorname{GF}(q)$. The automorphism group $\operatorname{Aut}(\mathrm{C})$ is said to be $t$-transitive if for every pair of $t$-element ordered sets of coordinates, there is an element $D P \gamma$ of the automorphism group $\operatorname{Aut}(\mathrm{C})$ such that its permutation part $P$ sends the first set to the second set.

The next theorem gives a sufficient condition for a linear code to hold $t$-designs [14, p. 308].

Theorem 2. Let $\mathrm{C}$ be a linear code of length $n$ over $\mathrm{GF}(q)$ where $\operatorname{Aut}(\mathrm{C})$ is $t$-transitive. Then the codewords of any weight $i \geq t$ of $\mathrm{C}$ hold a t-design.

\subsection{The generalized Reed-Muller codes}

The codes of a family of 2-designs held in a class of affine-invariant codes that will be studied in this paper are in fact subcodes of the fourth-order generalized Reed-Muller ternary codes. Hence, we review these codes and some of their properties in this section.

Let $\ell$ be a positive integer with $1 \leq \ell<(q-1) m$. The $\ell$-th order punctured generalized ReedMuller code $\mathcal{R}_{q}(\ell, m)^{*}$ over $\mathrm{GF}(q)$ is the cyclic code of length $n=q^{m}-1$ with generator polynomial

$$
g(x)=\sum_{\substack{1 \leq j \leq n-1 \\ \mathrm{wt} q(j)<(q-1) m-\ell}}\left(x-\alpha^{j}\right),
$$

where $\alpha$ is a generator of $\operatorname{GF}\left(q^{m}\right)^{*}$ [3]. Since $\operatorname{wt}_{q}(j)$ is a constant function on each $q$-cyclotomic coset modulo $n=q^{m}-1, g(x)$ is a polynomial over $\mathrm{GF}(q)$.

The parameters of the punctured generalized Reed-Muller code $\mathcal{R}_{q}(\ell, m)^{*}$ are known and summarized in the next theorem.

Theorem 3. [3] For any $\ell$ with $0 \leq \ell<(q-1) m, \mathcal{R}_{q}(\ell, m)^{*}$ is a cyclic code over $\mathrm{GF}(q)$ with length $n=q^{m}-1$, dimension

$$
\kappa=\sum_{i=0}^{\ell} \sum_{j=0}^{m}(-1)^{j}\left(\begin{array}{c}
m \\
j
\end{array}\right)\left(\begin{array}{c}
i-j q+m-1 \\
i-j q
\end{array}\right)
$$

and minimum weight $d=\left(q-\ell_{0}\right) q^{m-\ell_{1}-1}-1$, where $\ell=\ell_{1}(q-1)+\ell_{0}$ and $0 \leq \ell_{0}<q-1$. 
For $0 \leq \ell<m(q-1)$, the code $\left(\mathcal{R}_{q}(\ell, m)^{*}\right)^{\perp}$ is the cyclic code with generator polynomial

$$
g^{\perp}(x)=\sum_{\substack{0 \leq j \leq n-1 \\ \operatorname{wt}_{q}(j)<\ell}}\left(x-\alpha^{j}\right),
$$

where $\alpha$ is a generator of $\operatorname{GF}\left(q^{m}\right)^{*}$. In addition,

$$
\left(\mathcal{R}_{q}(\ell, m)^{*}\right)^{\perp}=(\mathrm{GF}(q) \overline{\mathbf{1}})^{\perp} \cap \mathcal{R}_{q}(m(q-1)-1-\ell, m)^{*},
$$

where $\overline{\mathbf{1}}$ is the all-one vector in $\operatorname{GF}(q)^{n}$ and $\operatorname{GF}(q) \overline{\mathbf{1}}$ denotes the code over $\operatorname{GF}(q)$ with length $n$ generated by $\overline{\mathbf{1}}$.

The parameters of the dual of the punctured generalized Reed-Muller code are summarized as follows [1, Section 5.4]. For $0 \leq \ell<m(q-1)$, the code $\left(\mathcal{R}_{q}(\ell, m)^{*}\right)^{\perp}$ has length $n=q^{m}-1$, dimension

$$
\kappa=n-\sum_{i=0}^{\ell} \sum_{j=0}^{m}(-1)^{j}\left(\begin{array}{c}
m \\
j
\end{array}\right)\left(\begin{array}{c}
i-j q+m-1 \\
i-j q
\end{array}\right) \text {, }
$$

and minimum weight

$$
d \geq\left(q-\ell_{0}^{\prime}\right) q^{m-\ell_{1}^{\prime}-1}
$$

where $m(q-1)-1-\ell=\ell_{1}^{\prime}(q-1)+\ell_{0}^{\prime}$ and $0 \leq \ell_{0}^{\prime}<q-1$.

The generalized Reed-Muller code $\mathcal{R}_{q}(\ell, m)$ is defined to be the extended code of $\mathcal{R}_{q}(\ell, m)^{*}$, and its parameters are given below [3]. Let $0 \leq \ell<q(m-1)$. Then the generalized Reed-Muller code $\mathcal{R}_{q}(\ell, m)$ has length $n=q^{m}$, dimension

$$
\kappa=\sum_{i=0}^{\ell} \sum_{j=0}^{m}(-1)^{j}\left(\begin{array}{c}
m \\
j
\end{array}\right)\left(\begin{array}{c}
i-j q+m-1 \\
i-j q
\end{array}\right)
$$

and minimum weight

$$
d=\left(q-\ell_{0}\right) q^{m-\ell_{1}-1}
$$

where $\ell=\ell_{1}(q-1)+\ell_{0}$ and $0 \leq \ell_{0}<q-1$.

Theorem 4. [3] Let $0 \leq \ell<q(m-1)$ and $\ell=\ell_{1}(q-1)+\ell_{0}$, where $0 \leq \ell_{0}<q-1$. The total number $A_{\left(q-\ell_{0}\right) q^{m-\ell_{1}-1}}$ of minimum weight codewords in $\mathcal{R}_{q}(\ell, m)$ is given by

$$
A_{\left(q-\ell_{0}\right) q^{m-\ell_{1}-1}}=(q-1) \frac{q^{\ell_{1}}\left(q^{m}-1\right)\left(q^{m-1}-1\right) \cdots\left(q^{\ell_{1}+1}-1\right)}{\left(q^{m-\ell_{1}}-1\right)\left(q^{m-\ell_{1}-1}-1\right) \cdots(q-1)} N_{\ell_{0}},
$$

where

$$
N_{\ell_{0}}= \begin{cases}1 & \text { if } \ell_{0}=0 \\
\left(\begin{array}{c}
q \\
\ell_{0}
\end{array}\right) \frac{q^{m-\ell_{1}}-1}{q-1} & \text { if } 0<\ell_{0}<q-1\end{cases}
$$


The generalized Reed-Muller codes $\mathcal{R}_{q}(\ell, m)$ can also be defined with a multivariate polynomial approach. The reader is referred to [3, Section 5.4] for details. For $\ell<(q-1) m$, it was shown in [3] that

$$
\mathcal{R}_{q}(\ell, m)^{\perp}=\mathcal{R}_{q}(m(q-1)-1-\ell, m) .
$$

The general affine group $\mathrm{GA}_{1}(\mathrm{GF}(q))$ is defined by

$$
\mathrm{GA}_{1}(\mathrm{GF}(q))=\left\{a x+b: a \in \mathrm{GF}(q)^{*}, b \in \mathrm{GF}(q)\right\},
$$

which acts on $\operatorname{GF}(q)$ doubly transitively [9, Section 1.7]. A linear code $C$ of length $q$ is said to be affine-invariant if $\mathrm{GA}_{1}(\mathrm{GF}(q))$ fixes $\mathrm{C}[6]$. For affine-invariant codes we use the elements of $\operatorname{GF}(q)$ to index the coordinates of their codewords.

Let $\ell$ be a positive integer with $1 \leq \ell<(q-1) m$. Then $\mathcal{R}_{q}(\ell, m)$ is affine-invariant, and the automorphism group $\operatorname{Aut}\left(\mathscr{R}_{q}(\ell, m)\right)$ is doubly transitive. These are well known facts about the generalized Reed-Muller codes $\mathcal{R}_{q}(\ell, m)$ [3, 9].

\section{Codes of designs held in a class of affine-invariant ternary codes}

Let $p$ be an odd prime and $m \geq 2$ be an integer. Define

$$
\mathrm{C}(m, p)=\left\{\left(\operatorname{Tr}_{p^{m} / p}\left(a x^{2}+b x\right)+h\right)_{x \in \mathrm{GF}\left(p^{m}\right)}: a, b \in \mathrm{GF}\left(p^{m}\right), h \in \mathrm{GF}(p)\right\} .
$$

Clearly, the code $\mathrm{C}(m, p)$ is affine-invariant, and holds 2-designs for each fixed nonzero weight (see [9, Section 6.2] and [11]). Let $d$ denote the minimum weight of $\mathrm{C}(m, p)$. Let $\mathbb{D}_{d}(\mathrm{C}(m, p))$ denote the design formed by the supports of the minimum weight codewords in $\mathrm{C}(m, p)$, and let $\mathrm{C}_{p}\left(\mathbb{D}_{d}(\mathrm{C}(m, p))\right)$ denote the linear code over $\mathrm{GF}(p)$ spanned by the incidence matrix of the design $\mathbb{D}_{d}(\mathrm{C}(m, p))$. An interesting problem is to determine the parameters of the code $\mathrm{C}_{p}\left(\mathbb{D}_{d}(\mathrm{C}(m, p))\right)$. This problem is hard to solve for general odd $p$, but is feasible in the case $p=3$.

Our objective of this paper is to compute the dimension of the code $C_{3}\left(\mathbb{D}_{d}(C(m, 3))\right)$, or equivalently, to determine the 3-rank of the incidence matrix of $\mathbb{D}_{d}(\mathrm{C}(m, 3))$, and to prove a lower bound on the minimum distance of the code $\mathrm{C}_{3}\left(\mathbb{D}_{d}(\mathrm{C}(m, 3))\right)$. We will also prove that $\mathrm{C}_{3}\left(\mathbb{D}_{d}(\mathrm{C}(m, 3))\right)$ is a subcode of the fourth-order generalized Reed-Muller ternary code.

In the rest of this section below, we fix $q=3^{m}$ and let $\operatorname{Tr}(x)$ denote the trace function from $\operatorname{GF}(q)$ to $\operatorname{GF}(3)$. The code $\mathrm{C}(m, 3)$ has four nonzero weights when $m$ is odd, and six nonzero weights when $m$ is even [11]. When $m$ is odd, the code $C(m, 3)$ has parameters $\left[3^{m}, 2 m+1,2 \times 3^{m-1}-3^{(m-1) / 2}\right]$, and the weight distribution of the code $\mathrm{C}(m, 3)$ is given in Table 1 [9]. The dual code has parameters $\left[3^{m}, 3^{m}-1-2 m, 5\right]$. Hence, the Assmus-Mattson theorem can also be employed to prove that the codewords of a fixed weight in $\mathrm{C}(m, 3)$ support a 2-design [10]. When $m \geq 3$ is odd, the minimum distance $d=2 \times 3^{m-1}-3^{(m-1) / 2}$, and the design $\mathbb{D}_{d}(\mathrm{C}(m, 3))$ has parameters $2-\left(3^{m}, d, d(d-1) / 2\right)$ [10]. We treat only the case that $m \geq 3$ is odd. At the end of this section, we will state the conclusions for even $m$, but will skip their proofs.

We first prove the following result.

Lemma 5. Let $m \geq 2$. Then $\overline{\mathbf{1}} \in \mathrm{C}(m, 3)^{\perp}$.

Proof. The desired conclusion follows from the definition of $\mathrm{C}(m, 3)$ and

$$
\sum_{x \in \mathrm{GF}(q)} x^{2}=0 \text { and } \sum_{x \in \mathrm{GF}(q)} x=0 .
$$


Table 1: Weight distribution of some ternary linear codes

\begin{tabular}{|l|l|}
\hline Weight $w$ & No. of codewords $A_{w}$ \\
\hline 0 & 1 \\
$2 \times 3^{m-1}-3^{(m-1) / 2}$ & $3^{2 m}-3^{m}$ \\
$2 \times 3^{m-1}$ & $\left(3^{m}+3\right)\left(3^{m}-1\right)$ \\
$2 \times 3^{m-1}+3^{(m-1) / 2}$ & $3^{2 m}-3^{m}$ \\
$3^{m}$ & 2 \\
\hline
\end{tabular}

We will nee the next auxiliary result.

Lemma 6. Let $m \geq 4$. For each $(a, h) \in \mathrm{GF}(q)^{*} \times \mathrm{GF}(3)$ define

$$
H(a, h)=\left\{x \in \operatorname{GF}(q): \operatorname{Tr}\left(a x^{2}\right)+h=0\right\} .
$$

For each nopnemptry set $S \subset \mathrm{GF}(q)$, define $\Delta(S)=\left\{s-s^{\prime}: s, s \in S\right\}$. Then $H(a, h)=-H(a, h)$ and $\Delta(H(a, h))=\mathrm{GF}(q)$.

Proof. For any $b \in \mathrm{GF}(q)$, it suffices to show there is a pair $(x, y) \in H(a, h) \times H(a, h)$ such that $y-x=b$. This conclusion is obvious for $b=0$, as $H(a, h)$ is not empty. Hence, we need to prove the conclusion for all $b \neq 0$. This is to prove that the system of equations

$$
\operatorname{Tr}\left(a x^{2}\right)+h=0 \text { and } \operatorname{Tr}\left(a(x+b)^{2}\right)+h=0
$$

has at least one solution $x \in \mathrm{GF}(q)$ for each nonzero $b$.

Let $N(a, b, h)$ denote the number of solutions $x \in \mathrm{GF}(q)$ of Equation (5) for $b \neq 0$. Let $\chi_{1}^{\prime}$ and $\chi_{1}$ denote the canonical characteristic of $\operatorname{GF}(3)$ and $\operatorname{GF}(q)$, respectively. Recall that $a \neq 0$ and $b \neq 0$. We have then

$$
\begin{array}{rl}
3^{2} & N(a, b, h) \\
= & \left.\sum_{x \in \mathrm{GF}(q)} \sum_{y_{1}, y_{2} \in \mathrm{GF}(3)} \chi_{1}^{\prime}\left\{y_{1}\left[\operatorname{Tr}\left(a x^{2}\right)+h\right]+y_{2}\left[\operatorname{Tr}\left(a(x+b)^{2}\right)+h\right)\right]\right\} \\
= & \sum_{y_{1}, y_{2} \in \mathrm{GF}(3)} \sum_{x \in \mathrm{GF}(q)} \chi_{1}^{\prime}\left(y_{1} h+y_{2} h\right) \chi_{1}\left[\left(y_{1}+y_{2}\right) a x^{2}+2 a b y_{2} x+y_{2} a b^{2}\right] \\
= & \sum_{x \in \mathrm{GF}(q)} 1+\sum_{y_{2} \in \mathrm{GF}(3)^{*} x \in \mathrm{GF}(q)} \chi_{1}^{\prime}(0) \chi_{1}\left(2 a b y_{2} x+y_{2} a b^{2}\right) \\
& +\sum_{y_{1} \neq-y_{2} x \in \mathrm{GF}(q)} \chi_{1}^{\prime}\left(y_{1} h+y_{2} h\right) \chi_{1}\left[\left(y_{1}+y_{2}\right) a x^{2}+2 a b y_{2} x+y_{2} a b^{2}\right] \\
= & 3^{m}+\sum_{y_{1} \neq-y_{2}} \sum_{x \in \mathrm{GF}(q)} \chi_{1}^{\prime}\left(y_{1} h+y_{2} h\right) \chi_{1}\left[\left(y_{1}+y_{2}\right) a x^{2}+2 a b y_{2} x+y_{2} a b^{2}\right] \\
= & 3^{m}+\sum_{y_{1} \neq-y_{2}} \chi_{1}^{\prime}\left(y_{1} h+y_{2} h\right) \sum_{x \in \mathrm{GF}(q)} \chi_{1}\left[\left(y_{1}+y_{2}\right) a x^{2}+2 a b y_{2} x+y_{2} a b^{2}\right]
\end{array}
$$


It then follows from the Weil bound on exponential sums in [18, p. 218] that

$$
\begin{aligned}
\left|3^{2} N(a, b, h)-3^{m}\right| & =\left|\sum_{y_{1} \neq-y_{2}} \chi_{1}^{\prime}\left(y_{1} h+y_{2} h\right) \sum_{x \in \mathrm{GF}(q)} \chi_{1}\left[\left(y_{1}+y_{2}\right) a x^{2}+2 a b y_{2} x+y_{2} a b^{2}\right]\right| \\
& \leq \sum_{y_{1} \neq-y_{2}}\left|\chi_{1}^{\prime}\left(y_{1} h+y_{2} h\right)\right|\left|\sum_{x \in \mathrm{GF}(q)} \chi_{1}\left[\left(y_{1}+y_{2}\right) a x^{2}+2 a b y_{2} x+y_{2} a b^{2}\right]\right| \\
& =\sum_{y_{1} \neq-y_{2}}\left|\sum_{x \in \mathrm{GF}(q)} \chi_{1}\left[\left(y_{1}+y_{2}\right) a x^{2}+2 a b y_{2} x+y_{2} a b^{2}\right]\right| \\
& \leq 6 \times 3^{m / 2} .
\end{aligned}
$$

Consequently,

$$
N(a, b, h) \geq 3^{m-2}-2 \times 3^{(m-2) / 2}>1
$$

for $m \geq 4$. This completes the proof.

Lemma 7. Let $m \geq 3$. Define by $Q(q)$ and $N(q)$ the set of nonzero squares and the set of nonsquares in $\mathrm{GF}(q)$. Then $\Delta(Q(q))=\mathrm{GF}(q)$ and $\Delta(N(q))=\mathrm{GF}(q)$.

Proof. It is known that $Q(q)$ and $N(q)$ are $(q,(q-1) / 2,(q-3) / 4)$ difference sets in $(\mathrm{GF}(q),+)$ for odd $m$, and $(q,(q-1) / 2,(q-5) / 4,(q-1) / 2)$ almost difference sets in $(\mathrm{GF}(q),+)$ for all even $m$ [8]. The desired conclusions then follow.

The proof of the following lemma is easy and omitted.

Lemma 8. For each $(a, b) \in \mathrm{GF}(q)^{*} \times \mathrm{GF}(q)$, there is exactly one $h \in \mathrm{GF}(3)$ such that the codeword $\mathbf{c}_{(a, b, h)}:=\left(\operatorname{Tr}\left(a x^{2}+b x\right)+h\right)_{x \in \mathrm{GF}(q)}$ has minimum Hamming weight d. Hence, the total number of minimum weight codewords is $q(q-1)$.

For each $(a, b) \in \mathrm{GF}(q)^{*} \times \mathrm{GF}(q)$, let $h(a, b)$ denote the unique element in $\operatorname{GF}(q)$ such that the codeword $\mathbf{c}_{(a, b, h)}:=\left(\operatorname{Tr}\left(a x^{2}+b x\right)+h(a, b)\right)_{x \in \mathrm{GF}(q}$ has the minimum weight. We use the elements of $\operatorname{GF}(q)$ to index the coordinates of the code $\mathrm{C}_{3}\left(\mathbb{D}_{d}(\mathrm{C}(m, 3))\right)$. We also use $\mathrm{GF}(q)$ as the point set of the design $\mathbb{D}_{d}(\mathrm{C}(m, 3))$.

For each $(a, b) \in \mathrm{GF}(q)^{*} \times \mathrm{GF}(q)$, define a vector

$$
\mathbf{g}(a, b, h(a, b))=\left(g(a, b, h(a, b))_{x}\right)_{x \in \mathrm{GF}(q)} \in \mathrm{GF}(3)^{q},
$$

where

$$
g(a, b, h(a, b))_{x}= \begin{cases}1 & \text { if } \operatorname{Tr}\left(a x^{2}+b x\right)+h(a, b) \neq 0 \\ 0 & \text { otherwise }\end{cases}
$$

By definition, each vector $\mathbf{g}(a, b, h(a, b))$ has minimum weight $d$, and the code $\mathrm{C}_{3}\left(\mathbb{D}_{d}(\mathrm{C}(m, 3))\right)$ is the linear subspace spanned by the vectors in the following set

$$
\left\{\mathbf{g}(a, b, h(a, b)),(a, b) \in \mathrm{GF}(q)^{*} \times \mathrm{GF}(q)\right\} .
$$


By definition, for each $x \in \mathrm{GF}(q)$ we have

$$
g(a, b, h(a, b))_{x}=\left(\operatorname{Tr}\left(a x^{2}+b x\right)+h(a, b)\right)^{2} .
$$

This expression will help us analyze the code $\mathrm{C}_{3}\left(\mathbb{D}_{d}(\mathrm{C}(m, 3))\right)$.

Recall that $\mathrm{C}(m, 3)$ is affine-invariant. Let $m$ be odd and let $h \in \mathrm{GF}(3)$ be the unique element such that the codeword $\left(\operatorname{Tr}\left(x^{2}\right)+h\right)_{x \in \mathrm{GF}(q)}$ has minimum weight in $\mathrm{C}(m, 3)$. It is easily seen that the set of all minimum weight codewords in $\mathrm{C}(m, 3)$ is given by

$$
\left\{ \pm\left(\operatorname{Tr}\left((a x+b)^{2}\right)+h\right)_{x \in \mathrm{GF}(q)}:(a, b) \in \mathrm{GF}(q)^{*} \times \mathrm{GF}(q)\right\}
$$

Consequently, we obtain the following lemma.

Lemma 9. Let $m \geq 3$ be odd. Then the code $\mathrm{C}_{3}\left(\mathbb{D}_{d}(\mathrm{C}(m, 3))\right)$ is linearly spanned by the vectors in following set:

$$
\left\{\left(\operatorname{Tr}\left((a x+b)^{2}\right)+h\right)_{x \in \mathrm{GF}(q)}^{2}:(a, b) \in \mathrm{GF}(q)^{*} \times \mathrm{GF}(q)\right\}
$$

In the following, we identify any vector $(f(x))_{x \in \mathrm{GF}(q)} \in \mathrm{GF}(3)^{q}$ with the function $f(x)$ from $\mathrm{GF}(q)$ to $\mathrm{GF}(3)$. This will simplify our discussions below. We are now ready to determine the dimension of the code $\mathrm{C}_{3}\left(\mathbb{D}_{d}(\mathrm{C}(m, 3))\right)$ and derive a lower bound on the minimum distance of the code.

Let $a \in \mathrm{GF}(q)^{*}$. Note that

$$
\begin{aligned}
& \left(\operatorname{Tr}\left((a x+b)^{2}\right)+h\right)^{2}=\operatorname{Tr}\left(a^{2} x^{2}\right)^{2}+\operatorname{Tr}(a b x)^{2}+\left(\operatorname{Tr}\left(b^{2}\right)+h\right)^{2}+ \\
& \quad \operatorname{Tr}\left(a^{2} x^{2}\right) \operatorname{Tr}(a b x)-\left(\operatorname{Tr}\left(b^{2}\right)+h\right) \operatorname{Tr}\left(a^{2} x^{2}\right)+\left(\operatorname{Tr}\left(b^{2}\right)+h\right) \operatorname{Tr}(a b x) \in \mathrm{C}_{3}\left(\mathbb{D}_{d}(\mathrm{C}(m, 3))\right) .
\end{aligned}
$$

Replacing $b$ with $-b$ in (7), we obtain

$$
\begin{aligned}
& \left(\operatorname{Tr}\left((a x-b)^{2}\right)+h\right)^{2}=\operatorname{Tr}\left(a^{2} x^{2}\right)^{2}+\operatorname{Tr}(a b x)^{2}+\left(\operatorname{Tr}\left(b^{2}\right)+h\right)^{2}- \\
& \quad \operatorname{Tr}\left(a^{2} x^{2}\right) \operatorname{Tr}(a b x)-\left(\operatorname{Tr}\left(b^{2}\right)+h\right) \operatorname{Tr}\left(a^{2} x^{2}\right)-\left(\operatorname{Tr}\left(b^{2}\right)+h\right) \operatorname{Tr}(a b x) \in \mathrm{C}_{3}\left(\mathbb{D}_{d}(\mathrm{C}(m, 3))\right) .
\end{aligned}
$$

Subtracting (8) from (7) yields

$$
\operatorname{Tr}\left(a^{2} x^{2}\right) \operatorname{Tr}(a b x)+\left(\operatorname{Tr}\left(b^{2}\right)+h\right) \operatorname{Tr}(a b x) \in \mathrm{C}_{3}\left(\mathbb{D}_{d}(\mathrm{C}(m, 3))\right),
$$

which is the same as

$$
\operatorname{Tr}\left(a^{2} x^{2}\right) \operatorname{Tr}(c x)+\left(\operatorname{Tr}\left((c / a)^{2}\right)+h\right) \operatorname{Tr}(c x) \in \mathrm{C}_{3}\left(\mathbb{D}_{d}(\mathrm{C}(m, 3))\right)
$$

for all $(a, c) \in \mathrm{GF}(q)^{*} \times \mathrm{GF}(q)$.

Adding (8) and (7) gives

$$
\operatorname{Tr}\left(a^{2} x^{2}\right)^{2}+\operatorname{Tr}(a b x)^{2}+\left(\operatorname{Tr}\left(b^{2}\right)+h\right)^{2}-\left(\operatorname{Tr}\left(b^{2}\right)+h\right) \operatorname{Tr}\left(a^{2} x^{2}\right) \in \mathrm{C}_{3}\left(\mathbb{D}_{d}(\mathrm{C}(m, 3))\right),
$$

which is the same as

$$
\operatorname{Tr}\left(a^{2} x^{2}\right)^{2}+\operatorname{Tr}(c x)^{2}+\left(\operatorname{Tr}\left((c / a)^{2}\right)+h\right)^{2}-\left(\operatorname{Tr}\left((c / a)^{2}\right)+h\right) \operatorname{Tr}\left(a^{2} x^{2}\right) \in \mathrm{C}_{3}\left(\mathbb{D}_{d}(\mathrm{C}(m, 3))\right)
$$

for all $(a, c) \in \mathrm{GF}(q)^{*} \times \mathrm{GF}(q)$. 
Lemma 10. Let $m \geq 3$ be an odd integer and $q=3^{m}$. Let $b, x \in \operatorname{GF}(q)$. Then the following hold.

1. $\sum_{a \in \mathrm{GF}(q)^{*}} \operatorname{Tr}\left(a^{2} x^{2}\right)^{2}=0$.

2. $\sum_{a \in \mathrm{GF}(q)} \operatorname{Tr}(a b x)^{2}=0$.

3. $\sum_{a \in \mathrm{GF}(q)} \operatorname{Tr}\left(a^{2} x^{2}\right)=0$.

Proof. If $x=0$, the conclusions are obvious. Next, let $x \neq 0$. Then,

$$
\begin{aligned}
\sum_{a \in \mathrm{GF}(q)^{*}} \operatorname{Tr}\left(a^{2} x^{2}\right)^{2} & =\sum_{a \in \mathrm{GF}(q)} \operatorname{Tr}\left(a^{2} x^{2}\right)^{2} \\
& =\sum_{a \in \mathrm{GF}(q)} \operatorname{Tr}\left(a^{2}\right)^{2} \\
& =\left|\left\{a \in \mathrm{GF}(q): \operatorname{Tr}\left(a^{2}\right) \neq 0\right\}\right| \bmod 3 \\
& =\mathrm{wt}\left(\left(\operatorname{Tr}\left(x^{2}\right)\right)_{x \in \mathrm{GF}(q)}\right) \bmod 3 \\
& =0,
\end{aligned}
$$

where the last equality follows from Table 3 .

If $b=0, \sum_{a \in \mathrm{GF}(q)^{*}} \operatorname{Tr}(a b x)^{2}=0$. Next, let $b \neq 0$. Then

$$
\begin{aligned}
\sum_{a \in \mathrm{GF}(q)^{*}} \operatorname{Tr}(a b x)^{2} & =\sum_{a \in \mathrm{GF}(q)} \operatorname{Tr}(a b x)^{2} \\
& =\sum_{a \in \mathrm{GF}(q)} \operatorname{Tr}(a)^{2} \\
& =|\{a \in \mathrm{GF}(q): \operatorname{Tr}(a) \neq 0\}| \bmod 3 \\
& =2 \cdot 3^{m-1} \bmod 3 \\
& =0 .
\end{aligned}
$$

Since $\sum_{a \in \mathrm{GF}(q)^{*}} a^{2}=0$, we have

$$
\sum_{a \in \mathrm{GF}(q)^{*}} \operatorname{Tr}\left(a^{2} x^{2}\right)=\sum_{a \in \mathrm{GF}(q)^{*}} \operatorname{Tr}\left(a^{2}\right)=\operatorname{Tr}\left(\sum_{a \in \mathrm{GF}(q)^{*}} a^{2}\right)=0 .
$$

This completes the proof.

Lemma 11. Let $m \geq 4$. We have $1 \in \mathrm{C}_{3}\left(\mathbb{D}_{d}(\mathrm{C}(m, 3))\right)$.

Proof. By (10), we have

$$
\sum_{a \in \operatorname{GF}(q)^{*}}\left(\operatorname{Tr}\left(a^{2} x^{2}\right)^{2}+\operatorname{Tr}(a b x)^{2}+\left(\operatorname{Tr}\left(b^{2}\right)+h\right)^{2}-\left(\operatorname{Tr}\left(b^{2}\right)+h\right) \operatorname{Tr}\left(a^{2} x^{2}\right)\right) \in \mathrm{C}_{3}\left(\mathbb{D}_{d}(\mathrm{C}(m, 3))\right) .
$$

It then follows from Lemma 10 that $\left(\operatorname{Tr}\left(b^{2}\right)+h\right)^{2} \in \mathrm{C}_{3}\left(\mathbb{D}_{d}(\mathrm{C}(m, 3))\right)$ for all $b \in \mathrm{GF}(q)$. One can find $b \in \operatorname{GF}(q)$ such that $\operatorname{Tr}\left(b^{2}\right)+h \neq 0$. The desired conclusion then follows.

Lemma 12. Let $m \geq 5$ be odd. For all $(a, c) \in \mathrm{GF}(q) \times \mathrm{GF}(q), \operatorname{Tr}\left(a x^{2}\right) \operatorname{Tr}(c x) \in \mathrm{C}_{3}\left(\mathbb{D}_{d}(\mathrm{C}(m, 3))\right)$. 
Proof. The conclusion is obvious for $a=0$ or $c=0$. We now assume that $a, c \in \operatorname{GF}(q)^{*}$. Recall the set $H\left(a^{-2}, h\right)$ defined in Lemma 6. By Lemma 6, there is a basis $\left\{c_{1}, c_{2}, \ldots, c_{m}\right\} \subset H\left(a^{-2}, h\right)$ of $\operatorname{GF}(q)$ over $\operatorname{GF}(3)$. It then follows from (9) that $\operatorname{Tr}\left(a^{2} x^{2}\right) \operatorname{Tr}\left(c_{i} x\right) \in \mathrm{C}_{3}\left(\mathbb{D}_{d}(\mathrm{C}(m, 3))\right)$ for all $i$. Consequently, $\operatorname{Tr}\left(a^{2} x^{2}\right) \operatorname{Tr}(c x) \in \mathrm{C}_{3}\left(\mathbb{D}_{d}(\mathrm{C}(m, 3))\right)$ for all $c \in \operatorname{GF}(q)$ and all $a \in \operatorname{GF}(q)^{*}$.

Recall the set $Q(q)$ defined in Lemma 7. For each fixed $c \in \mathrm{GF}(q)^{*}$, by Lemma 7 there is a basis $\left\{a_{1}, a_{2}, \ldots, a_{m}\right\} \subset Q(q)$ of $\mathrm{GF}(q)$ over $\mathrm{GF}(3)$ such that $\operatorname{Tr}\left(a_{i} x^{2}\right) \operatorname{Tr}(c x) \in \mathrm{C}_{3}\left(\mathbb{D}_{d}(\mathrm{C}(m, 3))\right)$. It then follows that $\operatorname{Tr}\left(a x^{2}\right) \operatorname{Tr}(c x) \in \mathrm{C}_{3}\left(\mathbb{D}_{d}(\mathrm{C}(m, 3))\right)$ for all $a \in \mathrm{GF}(q)$. The desired conclusion then follows.

Lemma 13. Let $m \geq 4$. For all $c \in \mathrm{GF}(q), \operatorname{Tr}(c x) \in \mathrm{C}_{3}\left(\mathbb{D}_{d}(\mathrm{C}(m, 3))\right)$.

Proof. By Lemma 12, $\operatorname{Tr}\left(a^{2} x^{2}\right) \operatorname{Tr}(c x) \in \mathrm{C}_{3}\left(\mathbb{D}_{d}(\mathrm{C}(m, 3))\right)$. It then follows from (9) that

$$
\left(\operatorname{Tr}\left((c / a)^{2}\right)+h\right) \operatorname{Tr}(c x) \in \mathrm{C}_{3}\left(\mathbb{D}_{d}(\mathrm{C}(m, 3))\right)
$$

for all $a \in \operatorname{GF}(q)^{*}$ and $c \in \operatorname{GF}(q)$. For each $c$ there is an $a \in \operatorname{GF}(q)$ such that $\operatorname{Tr}\left((c / a)^{2}\right)+h=1$. Consequently, $\operatorname{Tr}(c x) \in \mathrm{C}_{3}\left(\mathbb{D}_{d}(\mathrm{C}(m, 3))\right)$. This completes the proof.

Lemma 14. Let $m \geq 4$. For all $b_{1}, b_{2} \in \mathrm{GF}(q), \operatorname{Tr}\left(b_{1} x\right) \operatorname{Tr}\left(b_{2} x\right) \in \mathrm{C}_{3}\left(\mathbb{D}_{d}(\mathrm{C}(m, 3))\right)$.

Proof. One can find $a \in \operatorname{GF}(q)^{*}$ such that $\operatorname{Tr}\left(\frac{b_{1} b_{2}}{a^{2}}\right)=0$. Let $c_{1}=\frac{-b_{1}-b_{2}}{a}$ and $c_{2}=\frac{-b_{1}+b_{2}}{a}$. It follows from (10) and Lemma 11 that

$$
\operatorname{Tr}\left(a^{2} x^{2}\right)^{2}+\operatorname{Tr}\left(a c_{1} x\right)^{2}-\left(\operatorname{Tr}\left(c_{1}^{2}\right)+h\right) \operatorname{Tr}\left(a^{2} x^{2}\right) \in \mathrm{C}_{3}\left(\mathbb{D}_{d}(\mathrm{C}(m, 3))\right)
$$

and

$$
\operatorname{Tr}\left(a^{2} x^{2}\right)^{2}+\operatorname{Tr}\left(a c_{2} x\right)^{2}-\left(\operatorname{Tr}\left(c_{2}^{2}\right)+h\right) \operatorname{Tr}\left(a^{2} x^{2}\right) \in \mathrm{C}_{3}\left(\mathbb{D}_{d}(\mathrm{C}(m, 3))\right) .
$$

Subtracting (13) from (12) yields

$$
\left(\operatorname{Tr}\left(a\left(c_{1}+c_{2}\right) x\right)\right)\left(\operatorname{Tr}\left(a\left(c_{1}-c_{2}\right) x\right)\right)-\left(\operatorname{Tr}\left(c_{1}^{2}-c_{2}^{2}\right)\right) \operatorname{Tr}\left(a^{2} x^{2}\right) \in \mathrm{C}_{3}\left(\mathbb{D}_{d}(\mathrm{C}(m, 3))\right),
$$

which is the same as

$$
\operatorname{Tr}\left(b_{1} x\right) \operatorname{Tr}\left(b_{2} x\right)-\operatorname{Tr}\left(\frac{b_{1} b_{2}}{a^{2}}\right) \operatorname{Tr}\left(a^{2} x^{2}\right) \in \mathrm{C}_{3}\left(\mathbb{D}_{d}(\mathrm{C}(m, 3))\right) .
$$

The desired conclusion then follows from $\operatorname{Tr}\left(\frac{b_{1} b_{2}}{a^{2}}\right)=0$.

As a corollary of Lemma14, we have the following.

Lemma 15. Let $m \geq 4$. For all $a \in \operatorname{GF}(q), \operatorname{Tr}(a x)^{2} \in \mathrm{C}_{3}\left(\mathbb{D}_{d}(\mathrm{C}(m, 3))\right)$.

Lemma 16. Let $m \geq 4$. For all $a \in \operatorname{GF}(q), \operatorname{Tr}\left(a x^{2}\right) \in \mathrm{C}_{3}\left(\mathbb{D}_{d}(\mathrm{C}(m, 3))\right)$. 
Proof. By Lemma 15, $\operatorname{Tr}(c x)^{2} \in \mathrm{C}_{3}\left(\mathbb{D}_{d}(\mathrm{C}(m, 3))\right)$. It then follows from (11) that

$$
\operatorname{Tr}\left(a^{2} x^{2}\right)^{2}+\left(\operatorname{Tr}\left((c / a)^{2}\right)+h\right)^{2}-\left(\operatorname{Tr}\left((c / a)^{2}\right)+h\right) \operatorname{Tr}\left(a^{2} x^{2}\right) \in \mathrm{C}_{3}\left(\mathbb{D}_{d}(\mathrm{C}(m, 3))\right)
$$

for all $a \in \operatorname{GF}(q)^{*}$ and $c \in \operatorname{GF}(q)$. Choose $c_{1}$ and $c_{2}$ in $\operatorname{GF}(q)$ such that

$$
\operatorname{Tr}\left(\left(c_{1} / a\right)^{2}\right)+h=2 \text { and } \operatorname{Tr}\left(\left(c_{2} / a\right)^{2}\right)+h=1 .
$$

Plugging $c_{1}$ and $c_{2}$ into (14) yields

$$
\begin{aligned}
& \operatorname{Tr}\left(a^{2} x^{2}\right)^{2}+(2)^{2}-2 \operatorname{Tr}\left(a^{2} x^{2}\right) \in \mathrm{C}_{3}\left(\mathbb{D}_{d}(\mathrm{C}(m, 3))\right), \\
& \operatorname{Tr}\left(a^{2} x^{2}\right)^{2}+(1)^{2}-1 \operatorname{Tr}\left(a^{2} x^{2}\right) \in \mathrm{C}_{3}\left(\mathbb{D}_{d}(\mathrm{C}(m, 3))\right) .
\end{aligned}
$$

Taking the difference of the two functions above shows that $\operatorname{Tr}\left(a^{2} x^{2}\right) \in \mathrm{C}_{3}\left(\mathbb{D}_{d}(\mathrm{C}(m, 3))\right.$. The desired conclusion then follows from Lemma7.

Lemma 17. Let $m \geq 5$ be an odd integer. For all $b_{1}, b_{2} \in \mathrm{GF}(q), \operatorname{Tr}\left(b_{1} x^{2}\right) \operatorname{Tr}\left(b_{2} x^{2}\right) \in \mathrm{C}_{3}\left(\mathbb{D}_{d}(\mathrm{C}(m, 3))\right)$.

Proof. By Lemma15, $\operatorname{Tr}(c x)^{2} \in \mathrm{C}_{3}\left(\mathbb{D}_{d}(\mathrm{C}(m, 3))\right)$. By Lemma 16, $\operatorname{Tr}\left(a^{2} x^{2}\right) \in \mathrm{C}_{3}\left(\mathbb{D}_{d}(\mathrm{C}(m, 3))\right)$. It then follows from (11) and Lemma 11 that

$$
\operatorname{Tr}\left(a^{2} x^{2}\right)^{2}+\left(\operatorname{Tr}\left((c / a)^{2}\right)+h\right)^{2} \in \mathrm{C}_{3}\left(\mathbb{D}_{d}(\mathrm{C}(m, 3))\right)
$$

for all $a \in \mathrm{GF}(q)^{*}$ and $c \in \mathrm{GF}(q)$. It then follows Lemma 11 that $\operatorname{Tr}\left(a^{2} x^{2}\right)^{2} \in \mathrm{C}_{3}\left(\mathbb{D}_{d}(\mathrm{C}(m, 3))\right)$. Consequently,

$$
\operatorname{Tr}\left(a_{1}^{2} x^{2}\right)^{2}-\operatorname{Tr}\left(a_{2}^{2} x^{2}\right)^{2}=\operatorname{Tr}\left(\left(a_{1}^{2}+a_{2}^{2}\right) x^{2}\right) \operatorname{Tr}\left(\left(a_{1}^{2}-a_{2}^{2}\right) x^{2}\right) \in \mathrm{C}_{3}\left(\mathbb{D}_{d}(\mathrm{C}(m, 3))\right)
$$

for all $a_{1}$ and $a_{2}$. Since -1 is a quadratic non-residue, there are $\varepsilon_{1}, \varepsilon_{2} \in\{1,-1\}$ such that $\varepsilon_{1}\left(b_{1}+\right.$ $\left.b_{2}\right)$ and $\varepsilon_{2}\left(-b_{1}+b_{2}\right)$ are quadratic residue. Let $a_{1}, a_{2} \in \mathrm{GF}(q)$ such that $\varepsilon_{1}\left(b_{1}+b_{2}\right)=a_{1}^{2}$ and $\varepsilon_{2}\left(-b_{1}+b_{2}\right)=a_{2}^{2}$. Then, $a_{1}^{2}+a_{2}^{2}=\left(\varepsilon_{1}-\varepsilon_{2}\right) b_{1}+\left(\varepsilon_{1}+\varepsilon_{2}\right) b_{2}$ and $a_{1}^{2}-a_{2}^{2}=\left(\varepsilon_{1}+\varepsilon_{2}\right) b_{1}+\left(\varepsilon_{1}-\right.$ $\left.\varepsilon_{2}\right) b_{2}$. By (15), one has

$$
\operatorname{Tr}\left(\left(\left(\varepsilon_{1}-\varepsilon_{2}\right) b_{1}+\left(\varepsilon_{1}+\varepsilon_{2}\right) b_{2}\right) x^{2}\right) \operatorname{Tr}\left(\left(\left(\varepsilon_{1}+\varepsilon_{2}\right) b_{1}+\left(\varepsilon_{1}-\varepsilon_{2}\right) b_{2}\right) x^{2}\right) \in \mathrm{C}_{3}\left(\mathbb{D}_{d}(\mathrm{C}(m, 3))\right) .
$$

If $\varepsilon_{1}=\varepsilon_{2}, \operatorname{Tr}\left(\left(\left(\varepsilon_{1}+\varepsilon_{2}\right) b_{2}\right) x^{2}\right) \operatorname{Tr}\left(\left(\left(\varepsilon_{1}+\varepsilon_{2}\right) b_{1}\right) x^{2}\right)=\operatorname{Tr}\left(b_{1} x^{2}\right) \operatorname{Tr}\left(b_{2} x^{2}\right) \in \mathrm{C}_{3}\left(\mathbb{D}_{d}(\mathrm{C}(m, 3))\right)$. If $\varepsilon_{1}=-\varepsilon_{2}, \operatorname{Tr}\left(\left(\left(\varepsilon_{1}-\varepsilon_{2}\right) b_{2}\right) x^{2}\right) \operatorname{Tr}\left(\left(\left(\varepsilon_{1}-\varepsilon_{2}\right) b_{1}\right) x^{2}\right)=\operatorname{Tr}\left(b_{1} x^{2}\right) \operatorname{Tr}\left(b_{2} x^{2}\right) \in \mathrm{C}_{3}\left(\mathbb{D}_{d}(C(m, 3))\right)$. This completes the proof.

We are now ready to prove the following theorem.

Theorem 18. Let $m \geq 5$ be odd. Then $\mathrm{C}_{3}\left(\mathbb{D}_{d}(\mathrm{C}(m, 3))\right)$ is linearly spanned by the functions in the set

$$
\left\{\operatorname{Tr}\left(a x^{2}\right) \operatorname{Tr}\left(b x^{2}\right), \operatorname{Tr}\left(a x^{2}\right) \operatorname{Tr}(b x), \operatorname{Tr}(a x) \operatorname{Tr}(b x), \operatorname{Tr}(b x), 1: a, b \in \mathrm{GF}(q)\right\}
$$


Proof. By Lemma 17, $\operatorname{Tr}\left(a x^{2}\right) \operatorname{Tr}\left(b x^{2}\right) \in \mathrm{C}_{3}\left(\mathbb{D}_{d}(\mathrm{C}(m, 3))\right)$. It follows from Lemma 12 that all functions $\operatorname{Tr}\left(a x^{2}\right) \operatorname{Tr}(b x) \in \mathrm{C}_{3}\left(\mathbb{D}_{d}(\mathrm{C}(m, 3))\right)$. By Lemma 14, $\operatorname{Tr}(a x) \operatorname{Tr}(b x) \in \mathrm{C}_{3}\left(\mathbb{D}_{d}(\mathrm{C}(m, 3))\right)$. By Lemma 13, $\operatorname{Tr}(b x) \in \mathrm{C}_{3}\left(\mathbb{D}_{d}(\mathrm{C}(m, 3))\right)$. By Lemma 11, $1 \in \mathrm{C}_{3}\left(\mathbb{D}_{d}(\mathrm{C}(m, 3))\right)$. Consequently, $\mathrm{C}_{3}\left(\mathbb{D}_{d}(\mathrm{C}(m, 3))\right)$ contains the linear space spanned by the functions in the set in (16).

By (7), all the functions $\left(\operatorname{Tr}\left((a x+b)^{2}\right)+h\right)^{2}$ can be generated by the functions in the set in (16). This completes the proof.

For convenience of discussion below, we use $\langle S\rangle$ to denote the space over $\operatorname{GF}(3)$ linearly spanned by the functions from $\operatorname{GF}(q)$ to $\operatorname{GF}(3)$ in the set $S$. To achieve our objective, we have to prove the next lemmas.

Lemma 19. Let $m \geq 2$. Then

$$
\langle\{\operatorname{Tr}(a x) \operatorname{Tr}(b x): a, b \in \mathrm{GF}(q)\}\rangle=\left\{\sum_{j=0}^{m-1} \operatorname{Tr}\left(c_{j} x^{3^{j}+1}\right): c_{i} \in \mathrm{GF}(q)\right\} .
$$

Proof. Let $R$ denote the linear subspace in the right-hand side of (17). Let $\left\{\alpha^{3^{i}}: 0 \leq i \leq m-1\right\}$ be a normal basis of $\mathrm{GF}(q)$ over $\mathrm{GF}(3)$. Let $x=\sum_{i=0}^{m-1} x_{i} \alpha^{3^{i}}$, where $x_{i} \in \mathrm{GF}(3)$. Then $x^{3^{j}}=$ $\sum_{h=0}^{m-1} x_{(h-j) \bmod m} \alpha^{3^{h}}$ for all $0 \leq j \leq m-1$. We then deduce that

$$
x^{3^{j}+1}=\sum_{\ell=0}^{m-1}\left(\sum_{\substack{i+h=\ell(\bmod m) \\ 0 \leq i, h<m}} x_{i} x_{(h-j) \bmod m}\right) \alpha^{3^{\ell}}
$$

for all $0 \leq j \leq m-1$. It then follows that

$$
\alpha^{3^{u}} x^{3^{j}+1}=\sum_{\ell=0}^{m-1}\left(\sum_{\substack{i+h=\ell-u(\bmod m) \\ 0 \leq i, h<m}} x_{i} x_{(h-j) \bmod m}\right) \alpha^{3^{\ell}}
$$

and

$$
\operatorname{Tr}\left(\alpha^{3^{u}} x^{3^{j}+1}\right)=\operatorname{Tr}(\alpha) \sum_{\ell=0}^{m-1}\left(\sum_{\substack{i+h=\ell-u(\bmod m) \\ 0 \leq i, h<m}} x_{i} x_{(h-j) \bmod m}\right)
$$

for all $0 \leq j \leq m-1$ and $0 \leq u \leq m-1$. Hence, $R$ is linearly spanned by the following functions

$$
\sum_{\ell=0}^{m-1}\left(\sum_{\substack{i+h=\ell-u(\bmod m) \\ 0 \leq i, h<m}} x_{i} x_{(h-j) \bmod m}\right), 0 \leq j \leq m-1,0 \leq u \leq m-1 .
$$

Let $L$ denote the linear subspace in the left-hand side of (17). Note that

$$
\operatorname{Tr}\left(\alpha^{3^{u}} x\right)=\operatorname{Tr}(\alpha) \sum_{i=0}^{m-1} x_{(u-i) \bmod m}
$$


We have

$$
\operatorname{Tr}\left(\alpha^{3^{u}} x\right) \operatorname{Tr}\left(\alpha^{3^{v}} x\right)=\operatorname{Tr}(\alpha)^{2}\left(\sum_{i=0}^{m-1} x_{(u-i) \bmod m}\right)\left(\sum_{j=0}^{m-1} x_{(v-j) \bmod m}\right) .
$$

Hence, $L$ is linearly spanned by the following functions

$$
\left(\sum_{i=0}^{m-1} x_{(u-i) \bmod m}\right)\left(\sum_{j=0}^{m-1} x_{(v-j) \bmod m}\right), 0 \leq u \leq m-1,0 \leq v \leq m-1 .
$$

It can be verified that the set of functions in (19) is the same as the set of functions in (18). It then follows that $R=L$.

Lemma 20. Let $m \geq 2$. Then

$$
\left\langle\left\{\operatorname{Tr}\left(a x^{2}\right) \operatorname{Tr}(b x): a, b \in \mathrm{GF}(q)\right\}\right\rangle=\left\{\sum_{j=0}^{m-1} \operatorname{Tr}\left(c_{j} x^{2 \times 3^{j}+1}\right): c_{i} \in \mathrm{GF}(q)\right\} .
$$

Proof. The proof is similar to that of Lemma 19 and is omitted.

Lemma 21. Let $m \geq 2$. Then

$$
\left\langle\left\{\operatorname{Tr}\left(a x^{2}\right) \operatorname{Tr}\left(b x^{2}\right): a, b \in \mathrm{GF}(q)\right\}\right\rangle=\left\{\sum_{j=0}^{m-1} \operatorname{Tr}\left(c_{j} x^{2 \times 3^{j}+2}\right): c_{i} \in \mathrm{GF}(q)\right\} .
$$

Proof. The proof is similar to that of Lemma 19 and is omitted.

We have now the following trace representation of the code $\mathrm{C}_{3}\left(\mathbb{D}_{d}(\mathrm{C}(m, 3))\right)$.

Theorem 22. Let $m \geq 4$ be odd. Then $\mathrm{C}_{3}\left(\mathbb{D}_{d}(\mathrm{C}(m, 3))\right)$ is given by

$$
\left\{\sum_{i=0}^{m-1} \operatorname{Tr}\left(a_{i} x^{2 \cdot 3^{i}+2}\right)+\sum_{j=0}^{m-1} \operatorname{Tr}\left(b_{j} x^{2 \cdot 3^{j}+1}\right)+\sum_{\ell=0}^{m-1} \operatorname{Tr}\left(c_{\ell} x^{3^{\ell}+1}\right)+u: a_{i}, b_{j}, c_{\ell} \in \mathrm{GF}(q), u \in \mathrm{GF}(3)\right\} .
$$

Proof. Note that

$$
\left\{\operatorname{Tr}\left(b_{0} x^{2 \cdot 3^{0}+1}\right): b_{0} \in \mathrm{GF}(q)\right\}=\{\operatorname{Tr}(b x): b \in \mathrm{GF}(q)\} .
$$

The desired conclusion then follows from Theorem 18, Lemmas 19, 20, and 21

We are now ready to prove the following main result of this section.

Theorem 23. For each odd $m \geq 5$, we have

$$
\operatorname{dim}\left(\mathrm{C}_{3}\left(\mathbb{D}_{d}(\mathrm{C}(m, 3))\right)\right)=2 m^{2}+1
$$

and the minimum distance $d\left(\mathrm{C}_{3}\left(\mathbb{D}_{d}(\mathrm{C}(m, 3))\right)\right)$ of the code $\mathrm{C}_{3}\left(\mathbb{D}_{d}(\mathrm{C}(m, 3))\right)$ is lower bounded by $3^{m-2}$. 
Proof. Put $n=3^{m}-1$. Define

$$
\begin{aligned}
A & =\left\{-\left(2 \times 3^{i}+2\right): 0 \leq i \leq m-1\right\} \subset \mathbb{Z}_{n}, \\
A_{1} & =\left\{-\left(2 \times 3^{i}+2\right): 0 \leq i \leq(m-1) / 2\right\} \subset \mathbb{Z}_{n}, \\
B & =\left\{-\left(2 \times 3^{i}+1\right): 0 \leq i \leq m-1\right\} \subset \mathbb{Z}_{n}, \\
C & =\left\{-\left(3^{i}+1\right): 0 \leq i \leq m-1\right\} \subset \mathbb{Z}_{n}, \\
C_{1} & =\left\{-\left(3^{i}+1\right): 0 \leq i \leq(m-1) / 2\right\} \subset \mathbb{Z}_{n} .
\end{aligned}
$$

Put $J=A \cup B \cup C$. Let $\beta$ be a primitive element of $\operatorname{GF}(q)$. Denote by $\mathbb{M}_{i}(x)$ the minimal polynomial of $\beta^{i}$ over GF(3). Define

$$
H(x)=\operatorname{lcm}\left(\mathbb{M}_{i}(x): i \in J\right\},
$$

where $1 \mathrm{~cm}$ denotes the least common multiple of a set of polynomials. Let $\mathrm{C}_{3}^{m}$ denote the cyclic code over GF(3) of length $n$ with parity-check polynomial $H(x)$. By the Delsarte Theorem [7] and Theorem 22, $\mathrm{C}_{3}\left(\mathbb{D}_{d}(\mathrm{C}(m, 3))\right)$ is permutation-equivalent to the augmented code of the extended code of $\mathrm{C}_{3}^{m}$. As a result,

$$
\operatorname{dim}\left(C_{3}\left(\mathbb{D}_{d}(C(m, 3))\right)\right)=\operatorname{dim}\left(C_{3}^{m}\right)+1 .
$$

Below we compute the dimension of $\mathrm{C}_{3}^{m}$.

For each $i \in \mathbb{Z}_{n}$, the cyclotomic coset modulo $n$ containing $i$ is defined by

$$
S_{i}=\left\{i 3^{j} \bmod n: 0 \leq j \leq m-1\right\} \subset \mathbb{Z}_{n} .
$$

The dimension of $\mathrm{C}_{3}^{m}$ is given as

$$
\operatorname{dim}\left(\mathrm{C}_{3}^{m}\right)=\left|\bigcup_{i \in A \cup B \cup C} S_{i}\right|
$$

The following statements can be verified:

- $\left|S_{i}\right|=m$ for all $i \in J$.

- $S_{i} \cap S_{j}=\emptyset$ for all pairs of distinct $i$ and $j$ in $B$.

- $S_{i} \cap S_{j}=\emptyset$ for all pairs of distinct $i$ and $j$ in $A_{1}$, and every element in $A \backslash A_{1}$ is contained in some $S_{i}$ for $i \in A_{1}$.

- $S_{i} \cap S_{j}=\emptyset$ for all pairs of distinct $i$ and $j$ in $C_{1}$, and every element in $C \backslash C_{1}$ is contained in some $S_{i}$ for $i \in C_{1}$.

- $S_{i} \cap S_{j}=\emptyset$ for all $i \in B$ and $j$ in $A_{1}$.

- $S_{i} \cap S_{j}=\emptyset$ for all $i \in B$ and $j$ in $C_{1}$.

- $S_{i} \cap S_{j}=\emptyset$ for all $i \in A_{1}$ and $j$ in $C_{1}$, except $(i, j)=(0,1)$, in which case the two cosets are the same. 
We then deduce that

$$
\operatorname{dim}\left(C_{3}^{m}\right)=\left(\left|A_{1}\right|+\left|C_{1}\right|-1+|B|\right) m=\left(\frac{m+1}{2}+\frac{m+1}{2}-1+m\right) m=2 m^{2} .
$$

The desired conclusion on the dimension of $\mathrm{C}_{3}\left(\mathbb{D}_{d}(\mathrm{C}(m, 3))\right)$ then follows.

By Theorem 18 the code $\mathrm{C}_{3}\left(\mathbb{D}_{d}(\mathrm{C}(m, 3))\right)$ is a subcode of the fourth-order generalized ReedMuller code $\mathcal{R}_{3}(4, m)$. By Theorem 4 , the fourth-order generalised Reed-Muller code $\mathcal{R}_{3}(4, m)$ has dimension

$$
\kappa=\sum_{i=0}^{4} \sum_{j=0}^{m}(-1)^{j}\left(\begin{array}{c}
m \\
j
\end{array}\right)\left(\begin{array}{c}
i-3 j+m-1 \\
i-3 j
\end{array}\right)=\left(\begin{array}{c}
m+3 \\
4
\end{array}\right)+\left(\begin{array}{c}
m+2 \\
3
\end{array}\right)-\frac{(m-1) m}{2}+1>2 m^{2}+1
$$

and minimum distance $3^{m-2}$. The lower bound on the code $\mathrm{C}_{3}\left(\mathbb{D}_{d}(\mathrm{C}(m, 3))\right)$ then follows.

The lower bound $3^{m-2}$ on the minimum distance of $\mathrm{C}_{3}\left(\mathbb{D}_{d}(\mathrm{C}(m, 3))\right)$ is not very tight. It would be nice if the following open problem can be settled.

Open Problem 24. Determine the minimum distance of the ternary code $\mathrm{C}_{3}\left(\mathbb{D}_{d}(\mathrm{C}(m, 3))\right)$.

We remark that the conclusions of Theorem 23 are also true for even $m \geq 6$. The proofs of the lemmas and theorems for odd $m$ can be modified slightly to prove the conclusions of Theorem 23 for even $m$. The details are left to the reader. For $m \in\{2,3,4\}$, the parameters of $C_{3}\left(\mathbb{D}_{d}(C(m, 3))\right)$ are given in the following example. The example indicates that the code $\mathrm{C}_{3}\left(\mathbb{D}_{d}(\mathrm{C}(m, 3))\right)$ has good parameters. The dimensions of these codes agree with the formula $2 m^{2}+1$.

Example 25. Let $d$ denote the minimum weight of $\mathrm{C}(m, 3)$. The parameters of the code $\mathrm{C}(m, 3)$ and $\mathrm{C}_{3}\left(\mathbb{D}_{d}(\mathrm{C}(m, 3))\right)$ for $m=2,3,4$ are listed below:

$$
\begin{array}{rrr}
m & \mathrm{C}(m, 3) & \mathrm{C}_{3}\left(\mathbb{D}_{d}(\mathrm{C}(m, 3))\right) \\
2 & {[9,5,4]} & {[9,9,1]} \\
3 & {[27,7,15]} & {[27,19,6]} \\
4 & {[81,9,48]} & {[81,33,21]}
\end{array}
$$

The ternary code $\mathrm{C}(3,3)$ is distance-optimal [12] and has weight distribution

$$
1+702 z^{15}+780 z^{18}+702 z^{21}+2 z^{27} \text {. }
$$

The ternary code $\mathrm{C}_{3}\left(\mathbb{D}_{d}(\mathrm{C}(3,3))\right)$ is also distance-optimal [12] $]$ and has weight distribution

$$
\begin{aligned}
& 1+5148 z^{6}+14742 z^{7}+84240 z^{8}+370500 z^{9}+1314144 z^{10}+4081428 z^{11}+ \\
& 10838880 z^{12}+25050870 z^{13}+49975380 z^{14}+87147918 z^{15}+129957048 z^{16}+ \\
& 168370488 z^{17}+187697640 z^{18}+177251490 z^{19}+141674832 z^{20}+94909698 z^{21}+ \\
& 51504336 z^{22}+22428900 z^{23}+7492680 z^{24}+1796418 z^{25}+273780 z^{26}+20906 z^{27} .
\end{aligned}
$$

The weight distributions of $\mathrm{C}(3,3)$ and $\mathrm{C}_{3}\left(\mathbb{D}_{d}(\mathrm{C}(3,3))\right)$ demonstrate a big difference between the two codes.

The following problem would be challenging.

Open Problem 26. Determine the parameters of the code $\mathrm{C}_{p}\left(\mathbb{D}_{d}(\mathrm{C}(m, p))\right)$ for odd $p>3$.

We point out that the code $\mathrm{C}_{3}\left(\mathbb{D}_{d}(\mathrm{C}(m, 3))\right)$ is affine-invariant, thus it holds 2 -designs. Therefore, the following open problem would be interesting.

Open Problem 27. Determine the parameters of the 2-designs held in the code $\mathrm{C}_{3}\left(\mathbb{D}_{d}(\mathrm{C}(m, 3))\right)$. 


\section{Summary and concluding remarks}

The contribution of this paper is the study of the ternary codes $\mathrm{C}_{3}\left(\mathbb{D}_{d}(\mathrm{C}(m, 3))\right)$ carried out in Section 3 , where the dimensions of the codes were determined, and a lower bound on the minimum distance of the codes was proved. We also proved that the codes $\mathrm{C}_{3}\left(\mathbb{D}_{d}(\mathrm{C}(m, 3))\right)$ are subcodes of the fourth-order generalized Reed-Muller ternary codes. This shows that the code $\mathrm{C}_{3}\left(\mathbb{D}_{d}(\mathrm{C}(m, 3))\right)$ is much more complicated than the original code $\mathrm{C}(m, 3)$, which is defined by quadratic functions $\operatorname{Tr}\left(a x^{2}+b x\right)+c$. The difference between the two codes $\mathrm{C}(m, 3)$ and $\mathrm{C}_{3}\left(\mathbb{D}_{d}(\mathrm{C}(m, 3))\right)$ is also seen in their dimensions. The codes $\mathrm{C}_{3}\left(\mathbb{D}_{d}(\mathrm{C}(m, 3))\right)$ are affine-invariant, hence the codeworfds of any nonzero weight support 2-designs.

Several open problems were presented in this paper. The reader is cordially invited to settle them. The $p$-rank of $t$-designs, i.e., the dimension of the corresponding codes, can be used to classify $t$-designs of certain type. For example, the 2-rank and 3-rank of Steiner triple and quadruple systems were intensively studied and employed for counting and classifying Steiner triple and quadruple systems [15], [16], [21], [24], [25], [27], [28], [29], [30].

\section{References}

[1] E. F. Assmus Jr., J. D. Key, Designs and Their Codes, Cambridge University Press, Cambridge, 1992.

[2] E. F. Assmus Jr., H. F. Mattson Jr., New 5-designs, J. Comb. Theory 6 (1969) 122-151.

[3] E. F. Assmus Jr., J. D. Key, Polynomial codes and finite geometries. In: Pless V.S., Huffman W.C. (eds.), Handbook of Coding Theory, vol. II, pp. 1269-1343. Elsevier, Amsterdam (1998).

[4] T. Beth, D. Jungnickel, H. Lenz, Design Theory, Cambridge University Press, Cambridge, 1999.

[5] P. V. Ceccherini, J. W. P. Hirschfeld, The dimension of projective geometry codes, Discrete Math. 107 (1992), 117-126.

[6] P. Charpin, Codes cycliques étendus affines-invariants et antichaînes d'un ensemble partiellement ordonné, Discrete Math. 80 (1990) 229-247.

[7] P. Delsarte, On subfield subcodes of modified Reed-Solomon codes, IEEE Trans. Inf. Theory 21(5) (1975), 575-576.

[8] C. Ding, Codes from Difference Sets, World Scientific, Singapore, 2015.

[9] C. Ding, Designs from Linear Codes, World Scientific, Singapore, 2018.

[10] C. Ding, C. Li, Infinite families of 2-designs and 3-designs from linear codes, Discrete Mathematics 340(10) (2017) 2415-2431.

[11] X. Du, R. Wang, C. Fan, Infinite families of 2-designs from a class of cyclic codes with two non-zeros, arXiv:1904.04242 [math.CO], 2019. 
[12] M. Grassl, Code Tables, http://www.codetables.de

[13] N. Hamada, On the $p$-rank of the incidence matrix of a balanced or partially balanced incomplete block design and its applications to error correcting codes, Hiroshima Math. J. 3 (1973), 153-226.

[14] W. C. Huffman, V. Pless, Fundamentals of Error-Correcting Codes, Cambridge University Press, Cambridge, 2003.

[15] D. Jungnickel, S. S. Magliveras, V. D. Tonchev, A. Wassermann, The classification of Steiner triple systems on 27 points with 3-rank 24, Designs, Codes, and Cryptography, 87 (2019), 831-839.

[16] D. Jungnickel and V. D. Tonchev, Counting Steiner triple systems with classical parameters and prescribed rank, J. Combin. Theory, Ser. A, 162 (2019), 10-33.

[17] G. T. Kennedy, V. Pless, A coding-theoretic approach to extending designs, Discrete Math. 142 (1995), 155-168.

[18] R. Lidl, H. Niederreiter, Finite Fields, Encyclopedia of Mathematics and Its Application 20, Cambridge University Press, Cambridge, 1997.

[19] T. Kasami, S. Lin, W. Peterson, Some results on cyclic codes which are invariant under the affine group and their applications, Inform. and Control 11 (1968), 475-496.

[20] F. J. MacWilliams, N. J. A. Sloane, The Theory of Error-Correcting Codes, North-Holland, Amsterdam, 1977.

[21] O. P. Osuna, There are 1239 Steiner triple systems ST S(31) of 2-rank 27, Designs, Codes and Cryptography 40(2) (2006), 187-190.

[22] V. D. Tonchev, Quasi-symmetric designs, codes, quadrics, and hyperplane sections, Geometriae Dedicata 48 (1993), 295-308.

[23] V. D. Tonchev, Codes and designs, in: Handbook of Coding Theory, Vol. II, V. S. Pless, and W. C. Huffman, (Editors), Elsevier, Amsterdam, 1998, pp. 1229-1268.

[24] V. D. Tonchev, A mass formula for Steiner triple systems $\operatorname{STS}\left(2^{n}-1\right)$ of 2-rank $2^{n}-n, J$. Combin. Theory, Ser. A, 95 (2001), 197-208.

[25] V. D. Tonchev, A formula for the number of Steiner quadruple systems on $2^{n}$ points of 2-rank $2^{n}-n$, Journal of Combinatorial Designs, 11 (2003), 260-274.

[26] V. D. Tonchev, Codes, in: Handbook of Combinatorial Designs, 2nd Edition, C. J. Colbourn, and J. H. Dinitz, (Editors), CRC Press, New York, 2007, pp. 677-701.

[27] D. V. Zinoviev, The number of Steiner triple systems $S\left(2^{m}-1,3,2\right)$ of rank $2^{m}-m+2$ over $\mathbb{F}_{2}$. Discr. Math. 339 (2016), 2727-2736. 
[28] V. A. Zinoviev, D. V. Zinoviev, Steiner triple systems $S\left(2^{m}-1,3,2\right)$ of rank $2^{m}-m+1$ over $\mathbb{F}_{2}$. Problems of Information Transmission 48 (2012), 102-126.

[29] V. A. Zinoviev, D. V. Zinoviev, Structure of Steiner triple systems $S\left(2^{m}-1,3,2\right)$ of rank $2^{m}-m+2$ over $\mathbb{F}_{2}$. Problems of Information Transmission 49 (2013), 232-248.

[30] V. A. Zinoviev, D. V. Zinoviev, Remark on "Steiner triple systems $S\left(2^{m}-1,3,2\right)$ of rank $2^{m}-m+1$ over $\mathbb{F}_{2}$ published in Probl. Peredachi Inf., 2012, no. 2," Problems of Information Transmission 49 (2013), 107-111. 